\title{
Quel potentiel de développement et quels consommateurs à l'international pour les vins rosés?
}

B. Eymard ${ }^{1, a}$, Brice Amato $^{2, b}$ et R.C. Delerins ${ }^{3}$

${ }^{1}$ Directeur Général, CIVP

${ }^{2}$ Responsable du service analyse des vignobles et marchés, CIVP, Conseil Interprofessionnel des Vins de Provence, Maison des Vins, RN 7 - CS 50002, 83460 Les Arcs sur Argens, France

${ }^{3}$ Food 2.0 Lab, Paris, France

\section{Un marché mondial du rosé en croissance}

La production et la consommation de rosé représentent 22 à 24 millions d'hl, soit $10 \%$ du volume de vins mondial. Le marché reste concentré.

5 pays représentent $75 \%$ de la production mondiale : le France, les Etats-Unis, l'Espagne, l'Italie et l'Afrique du Sud.

3 pays pèsent $60 \%$ de la consommation mondiale : la France, les Etats-Unis et l'Allemagne.
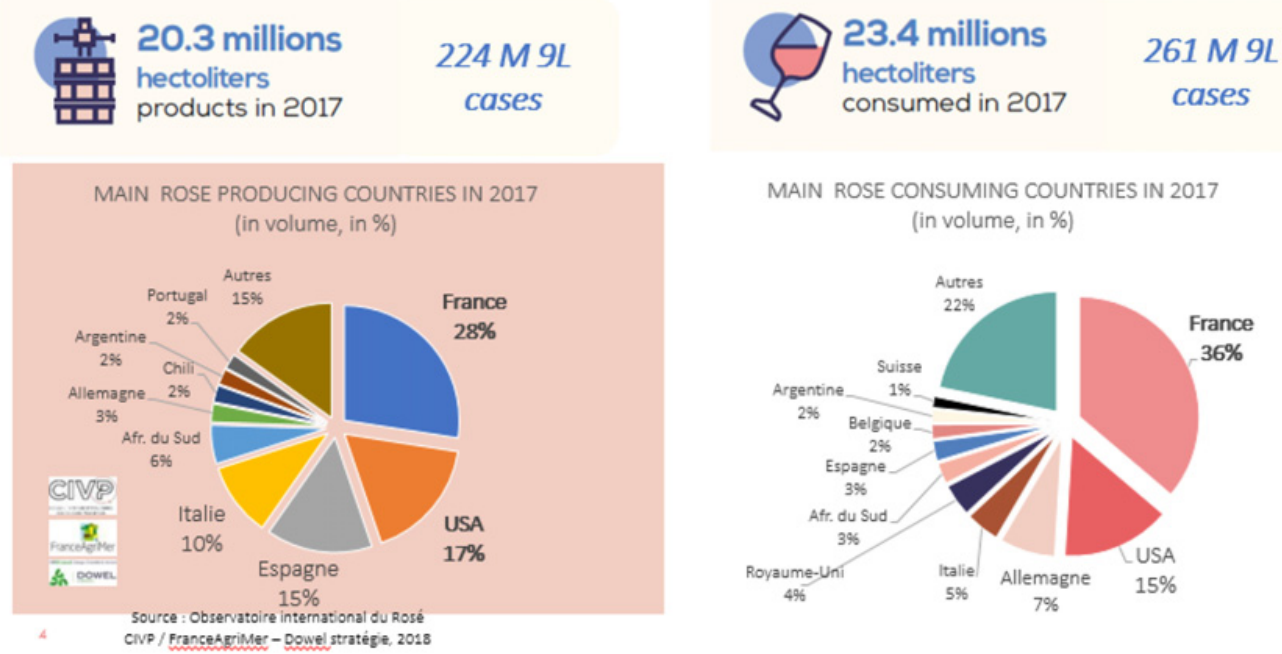

MAIN ROSE CONSUMING COUNTRIES IN 2017 (in volume, in \%)
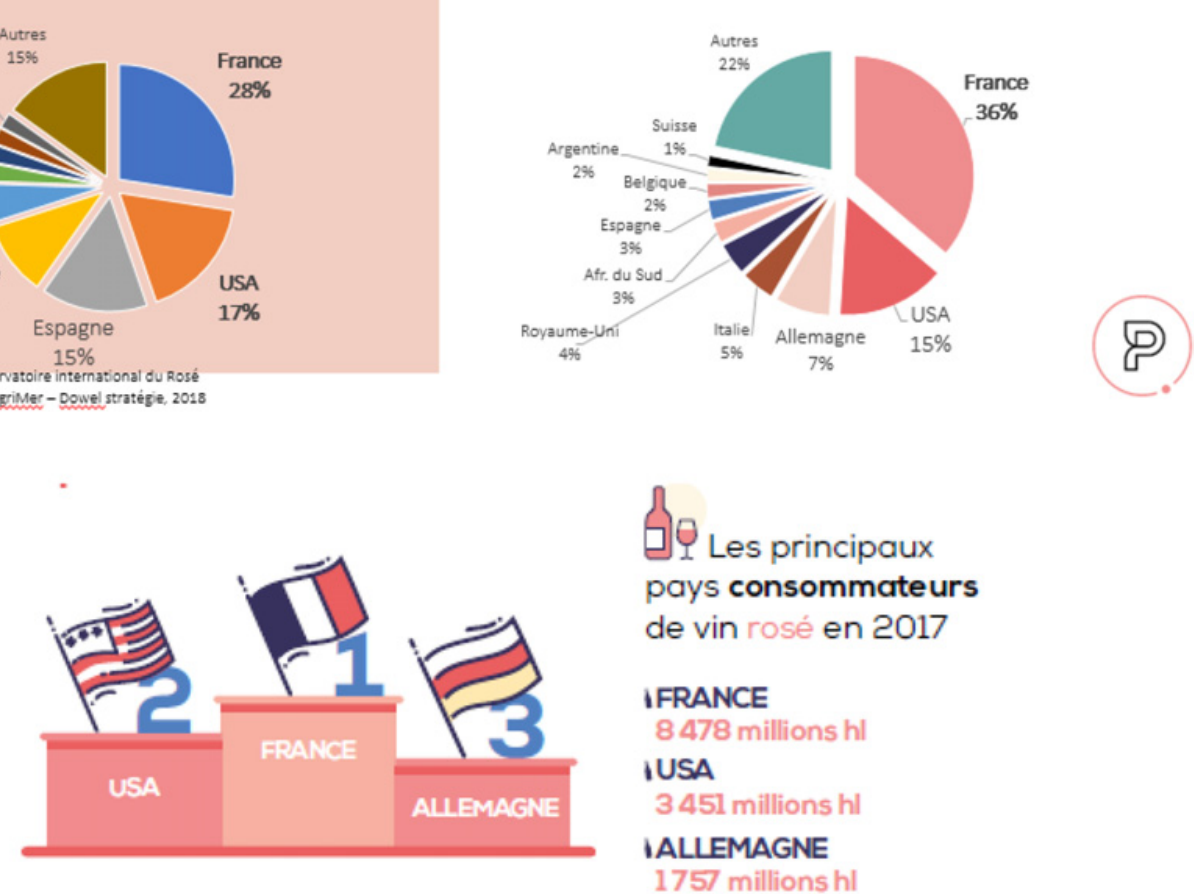

\footnotetext{
a e-mail: beymard@provencewines.com

b e-mail: bamato@provencewines.com
}

(C) The Authors, published by EDP Sciences. This is an Open Access article distributed under the terms of the Creative Commons Attribution License 4.0 (http://creativecommons.org/licenses/by/4.0/). 
Les échanges internationaux ont fortement progressé pour atteindre $40 \%$ du marché en 2017. Ils représentent ainsi environ 9 millions d'hL (soit $100 \mathrm{M}$ de caisses de 9L). Deux pays dominent fortement les échanges : l'Espagne est le principal exportateur de vins rosés vers la France qui est le plus gros importateur de vins rosés.

\section{Les principaux importateurs de vin rosé en 2003 (en \% du volume total des importations de vin rosé)}

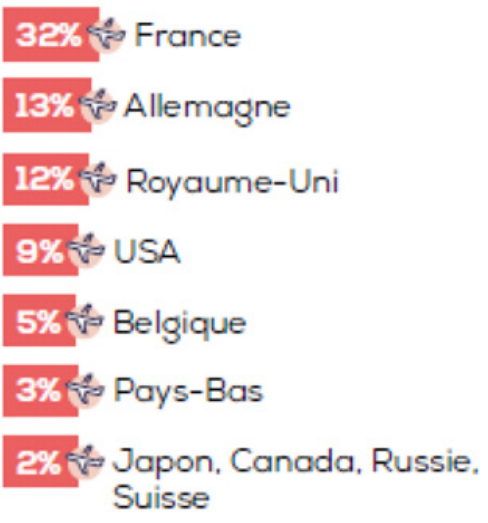

$18 \%$ Autres

\section{Les principaux exportateurs de vin rosé en 2017 \\ (en \% du volume totalde vin rosé exporté)}

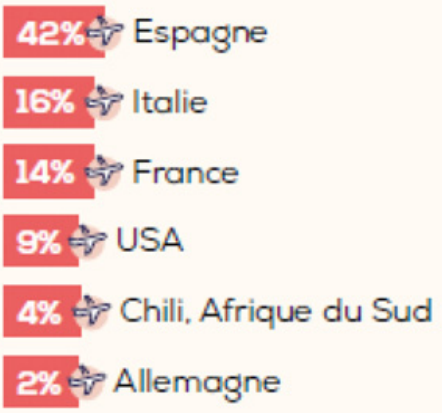

Flow of rosé wines in 2017

(Sources : Observatoire mondial du rosé, OIV)
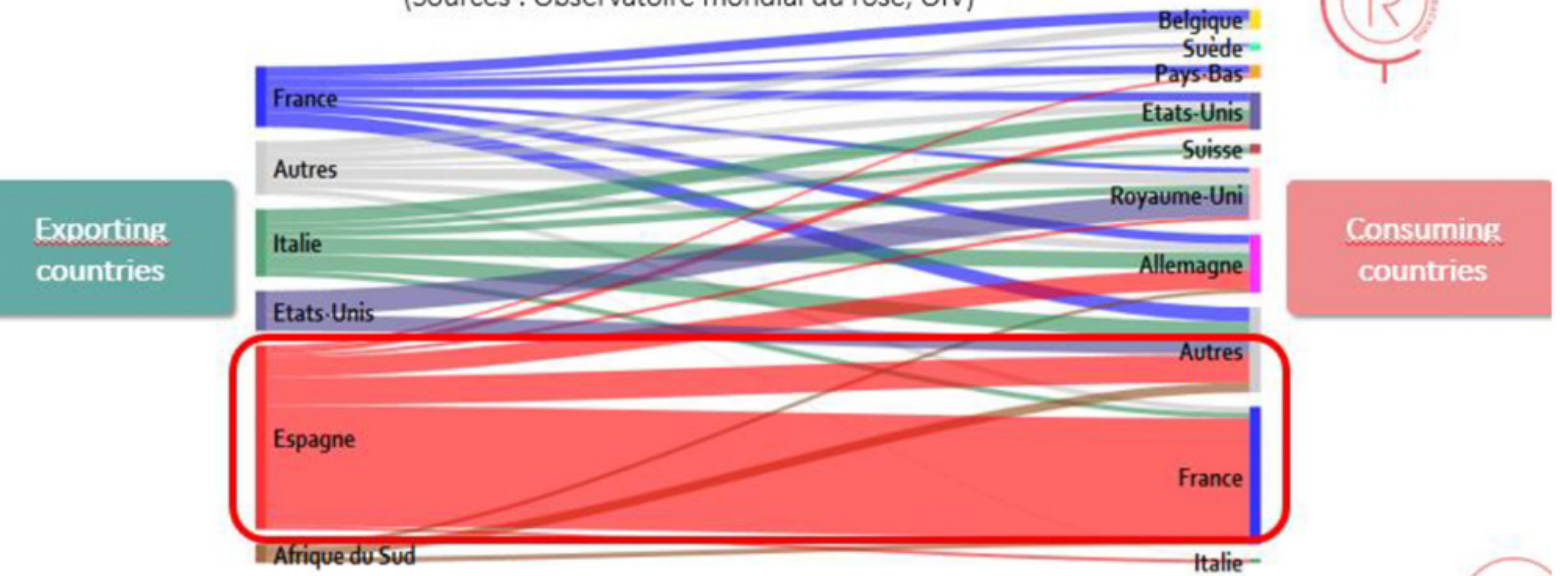

Le marché du rosé est en croissance continue au niveau international depuis 15 ans $(+30 \%$ en volume entre 2002 et 2017). De $8.4 \%$ en 2002, le vin rosé représente désormais plus de $10 \%$ de la consommation mondiale de vin. Une bouteille de vin sur 10 consommé dans le monde est donc aujourd'hui du rosé et c'est même une bouteille sur 3 en France (source : observatoire mondial du rosé, France Agrimer, CIVP, panel IRI). 

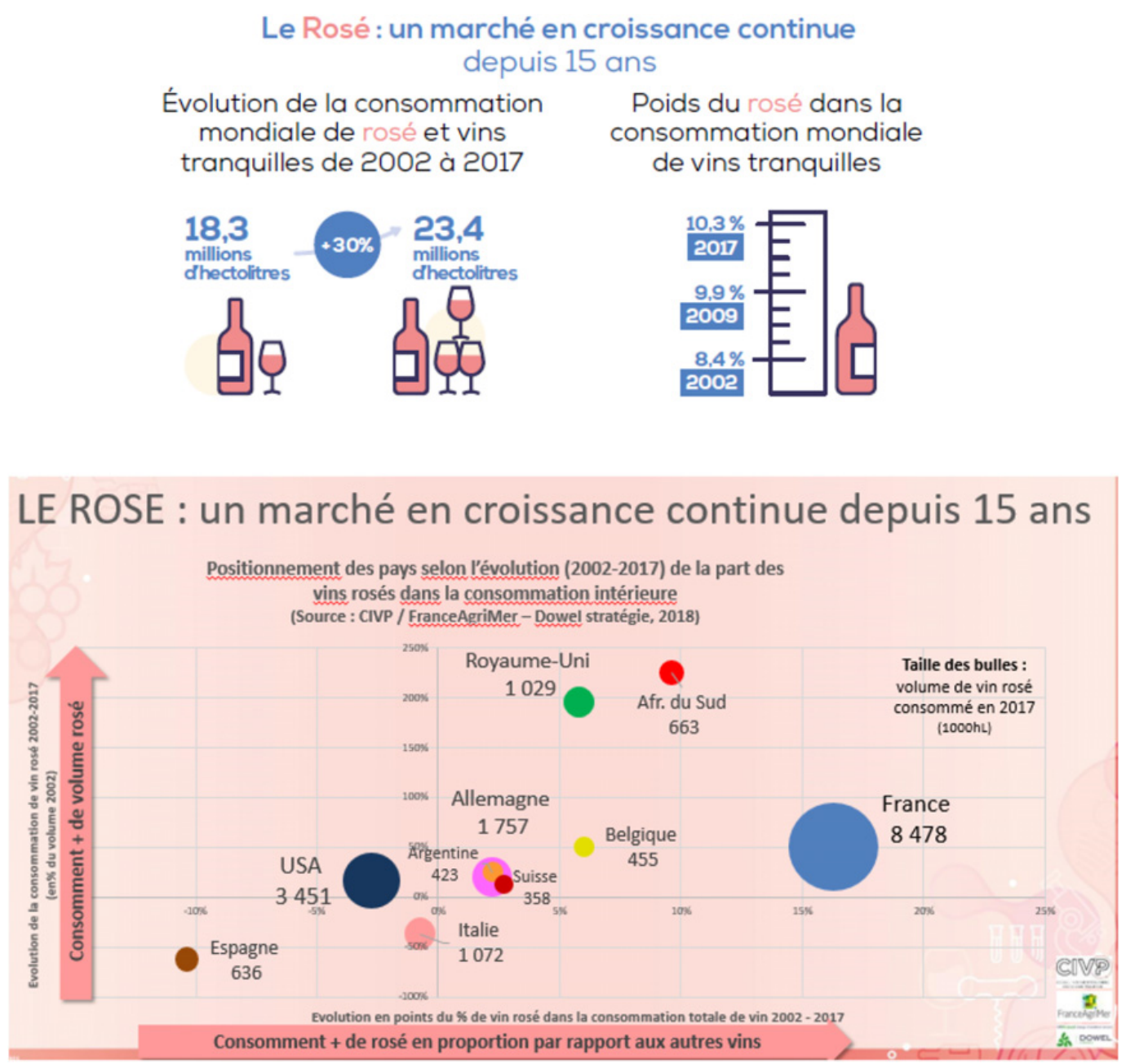

Ce développement s'est accéléré après 2010 avec notamment une demande importante en vins rosés secs et clairs.

L'observatoire des références en vente en magasin ou sur internet montre ainsi une diminution importante des rosés foncés entre 2013 (51\% de l'offre) et 2017 (31\%) au profit des vins rosés plus clairs. L'observation est la même pour le taux de sucre avec les vins de plus de $10 \mathrm{~g} / \mathrm{l}$ qui perdent plus de 10 points.

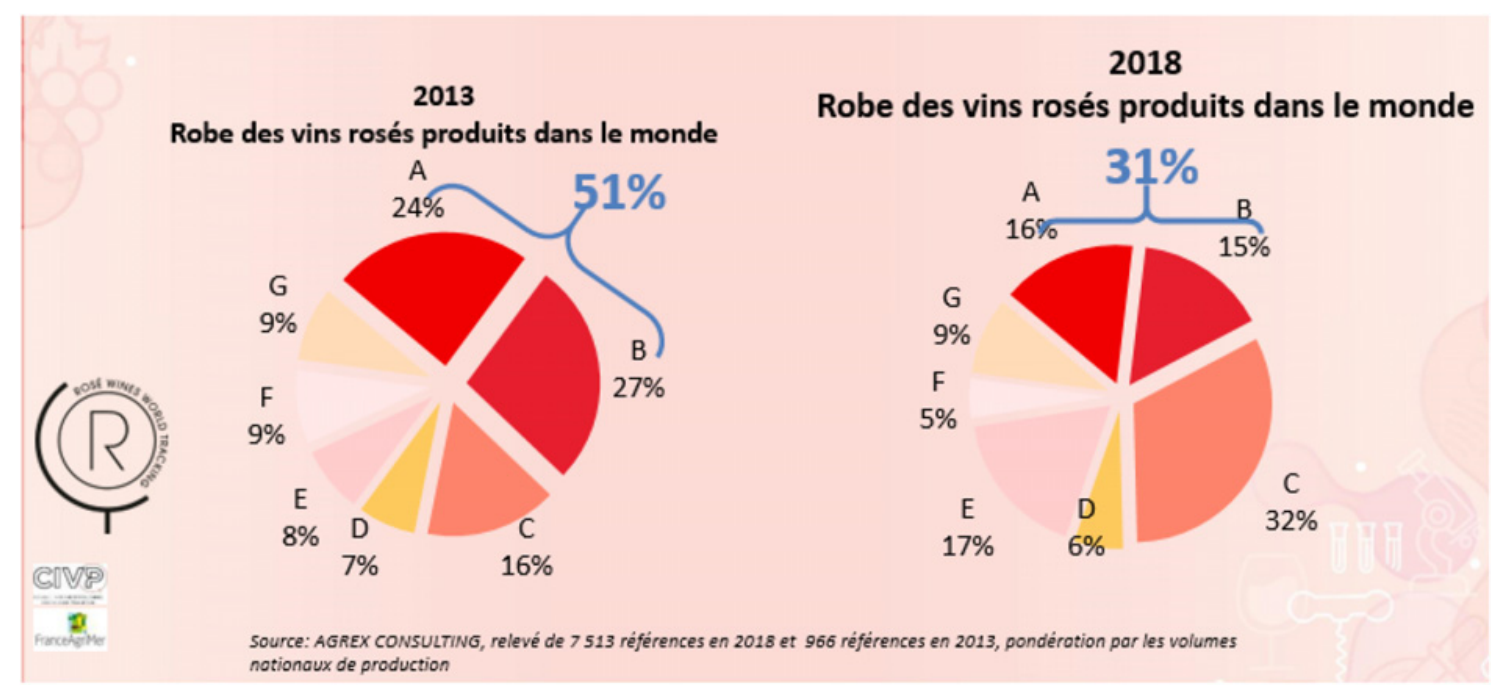




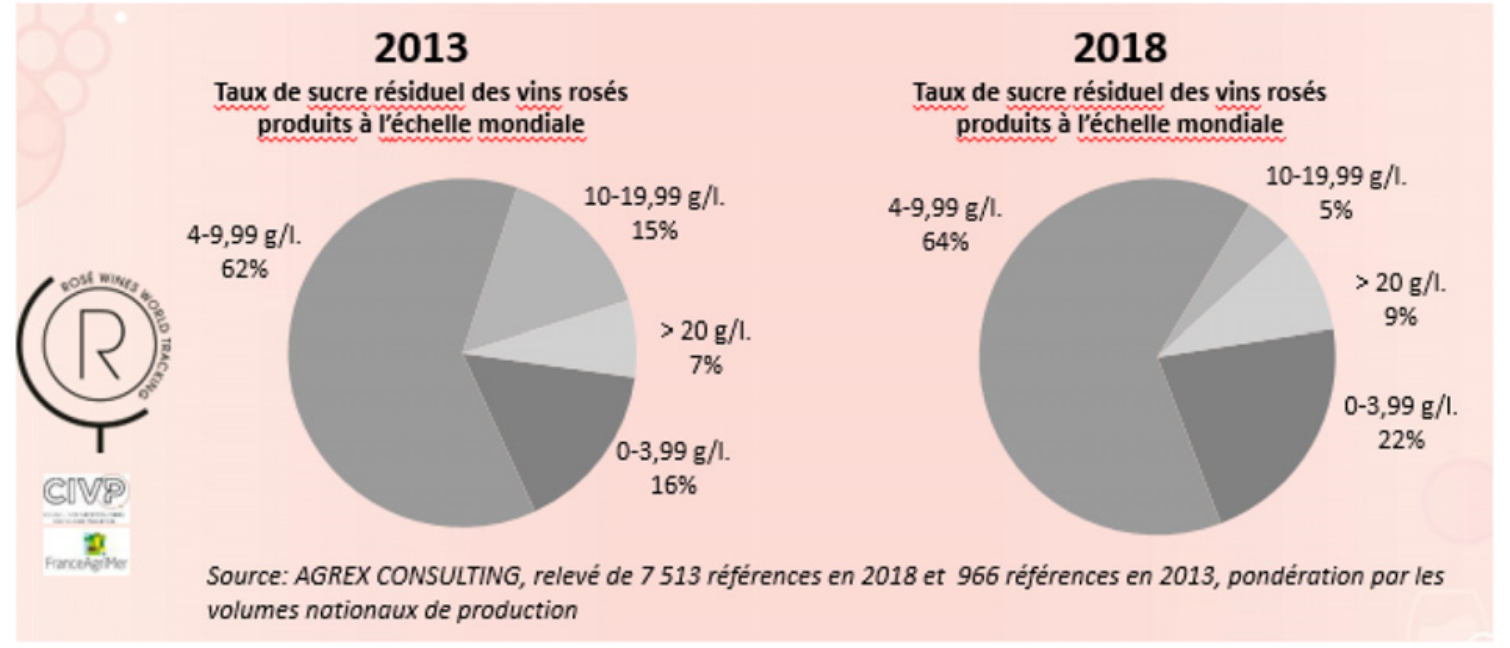

\section{Le marché mondial du rosé : Un marché émergent à potentiel}

Le marché du vin rosé est loin d'être mûr. Comme nous l'avons vu plus haut, il est dominé par la consommation en France (16 L/an/hab) et les exportations espagnoles ( $42 \%$ des expéditions mondiales en volume) mais il est encore émergent sur de nombreux pays.

Seulement 1/4 des 47 pays étudiés dans l'observatoire mondial du rosé ont une part de rosé consommé supérieure à $10 \%$. A titre d'exemple, aux USA, moteurs de la croissance du rosé ses dernières années, la consommation n'est que de $1.3 \mathrm{~L} / \mathrm{an} / \mathrm{hab}$ et seulement $16 \%$ des adultes boivent du rosé au moins une fois par mois. L'Asie est pour l'instant un territoire très peu demandeur de rosé du fait de la maturité des marchés de vins dans cette zone et d'habitudes de consommation encore peu favorables. En Chine, la consommation de rosé est estimée à moins de $0.01 \mathrm{~L} / \mathrm{an} / \mathrm{hab}$ et ne concernerait que $3 \%$ des adultes des grandes zones urbaines.

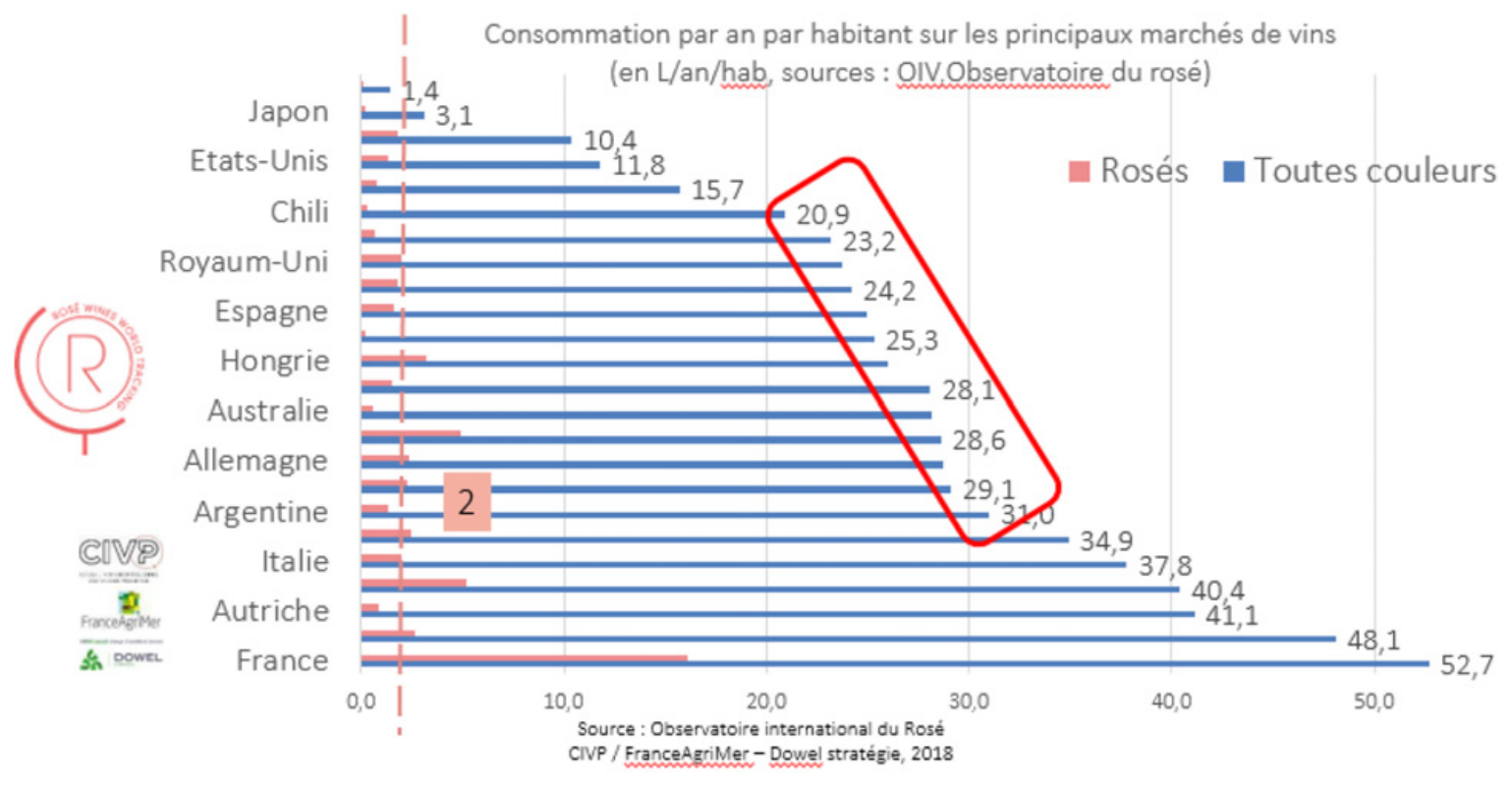




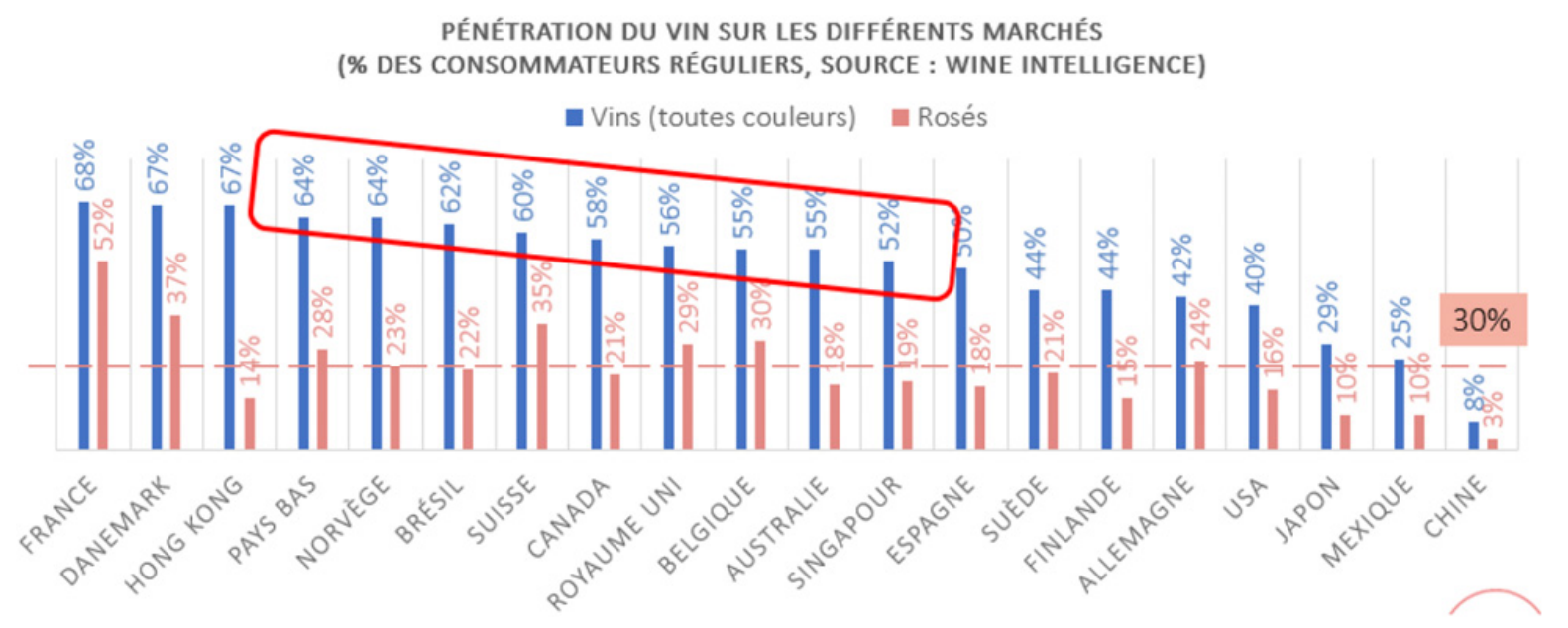

Le potentiel de développement des vins rosés dans les prochaines années est donc important. Les tendances actuelles ainsi que l'évolution structurelle des pays historiquement non consommateurs de vin permettent d'envisager une croissance du nombre de consommateurs et de la part de rosé consommés par ceux-ci sur une trentaine de pays différents, entrainant une diversification des marchés.

Nous avons comparé deux méthodes différentes d'estimation de l'évolution de la consommation de rosé dans les 15 prochaines années :

- La première a consisté à envisager une évolution des principaux marchés consommateurs de vins rosés vers une moyenne de part de marché de 10 à $15 \%$ de la consommation totale de vin et une consommation par an par habitant entre 2.5 et 4 litres/an/habitant

- La seconde est basée sur les évolutions passées du marché du rosé et l'étude prospective IWSR/Vinexpo 2018 et sur un étirement linéaire de ces tendances sur les principaux marchés.

(VOLUME * $1000 \mathrm{HL}$, SOURCE : ROSE WINES WORLD TRACKING)

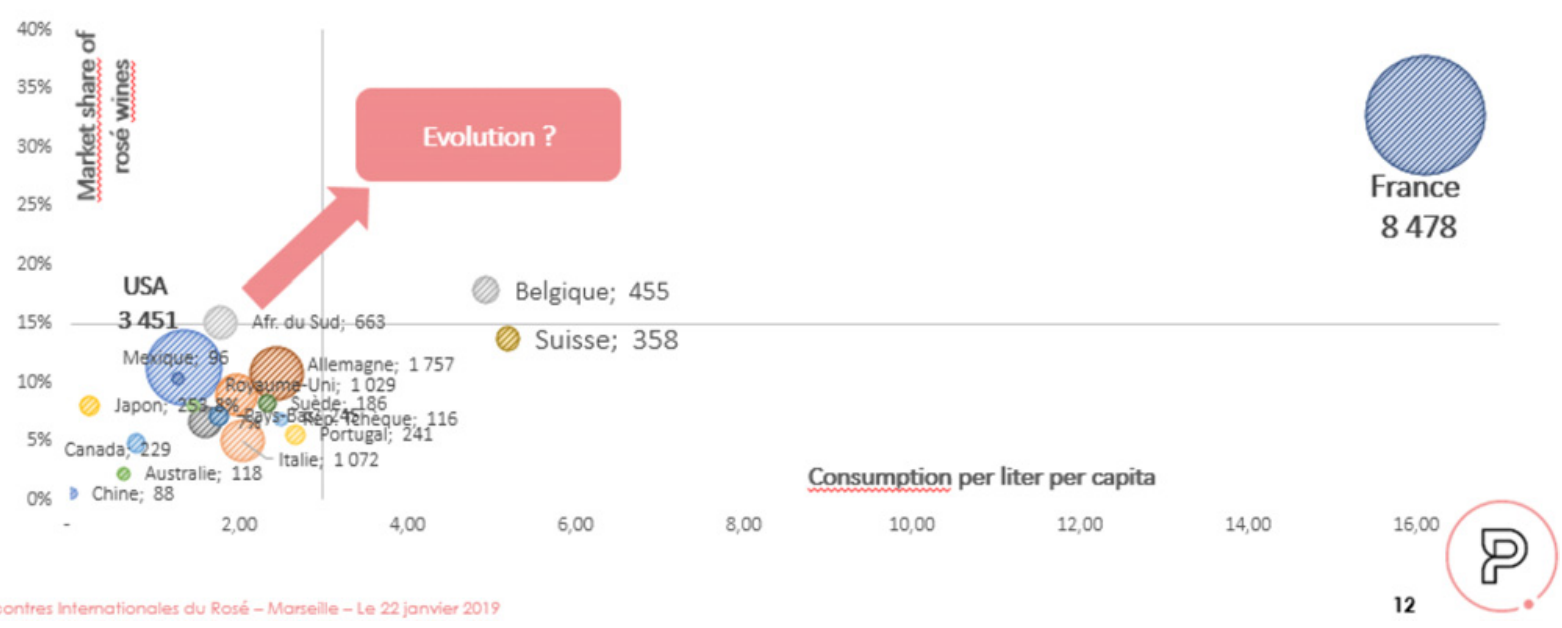

Les deux méthodes aboutissent globalement au même résultat. D'ici 2035, la consommation mondiale de rosé pourrait ainsi atteindre entre $\mathbf{2 8}$ et $\mathbf{3 0}$ millions d'hl avec notamment un développement en Amérique du Nord, en Asie et en Europe.

Cette évolution dépend bien sûr de nombreux facteurs avec en particulier deux interrogations: la capacité du «dry rosé» à se substituer en grande partie au «blush» aux USA et le potentiel de consommation sur les marchés asiatiques et en particulier la Chine qui, si les barrières culturelles sont levées, pourrait évoluer beaucoup plus brusquement et rapidement comme on a pu le voir sur les vins rouges. 
$28-30 \mathrm{Mhl}$

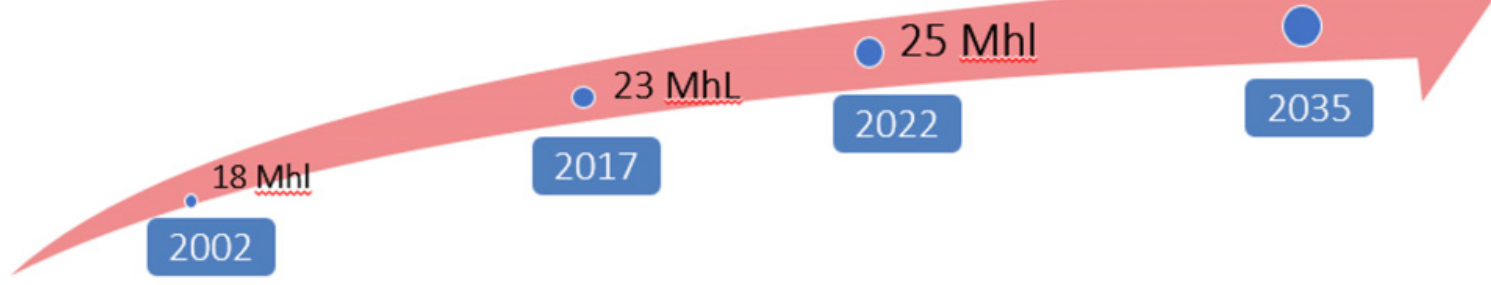

POTENTIAL GROWTH FOR THE ROSE MARKET BY AREAS

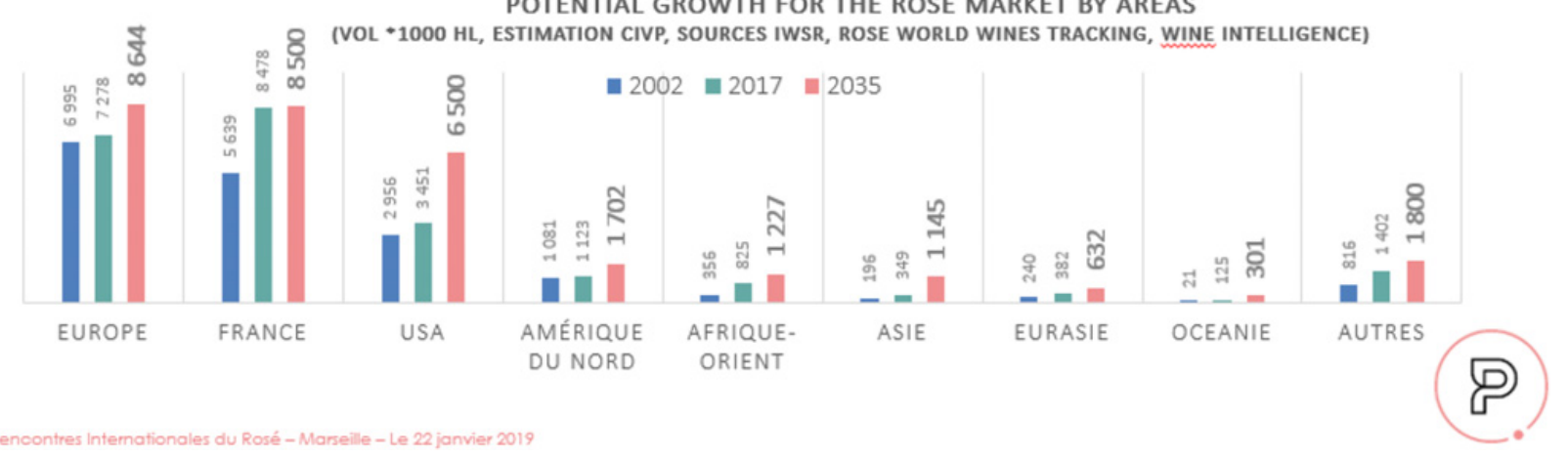

\section{Les vins de provence, référence sur le rosé}

Dans les 10 dernières années, les Vins de Provence ont bénéficié mais ont également été le moteur de la croissance des vins rosés au niveau mondial.

Le vignoble de Provence est depuis ses débuts spécialisé dans le rosé (90\% de la production).

\section{Revendications 2018 Vins de Provence \\ (CIVP d'après ODG en hl)}

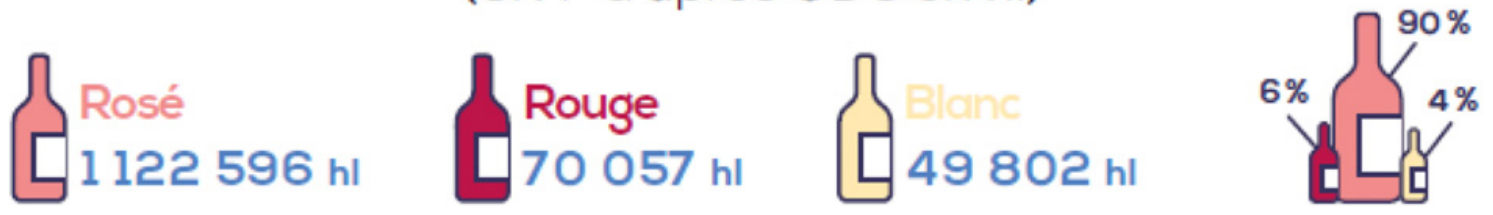

Avec une production de plus de 1.1 millions d'hL, les Côtes de Provence, Coteaux d'Aix en Provence et Coteaux Varois en Provence représentent $5 \%$ du rosé produits au niveau mondial et $42 \%$ des AOC française. C'est ainsi le premier vignoble mondial de rosé AOC.

Le marché des Vins de Provence a profondément changé en 10 ans. Les exportations de rosé qui pesaient $6 \%$ de la commercialisation en 2008 sont passées à $37 \%$ en 2018. La croissance des exportations portée par le marché US a ainsi été exponentielle ses dernières années : $+400 \%$ en volume et $+715 \%$ en valeur entre 2010 et 2018 (sources douanes françaises). 

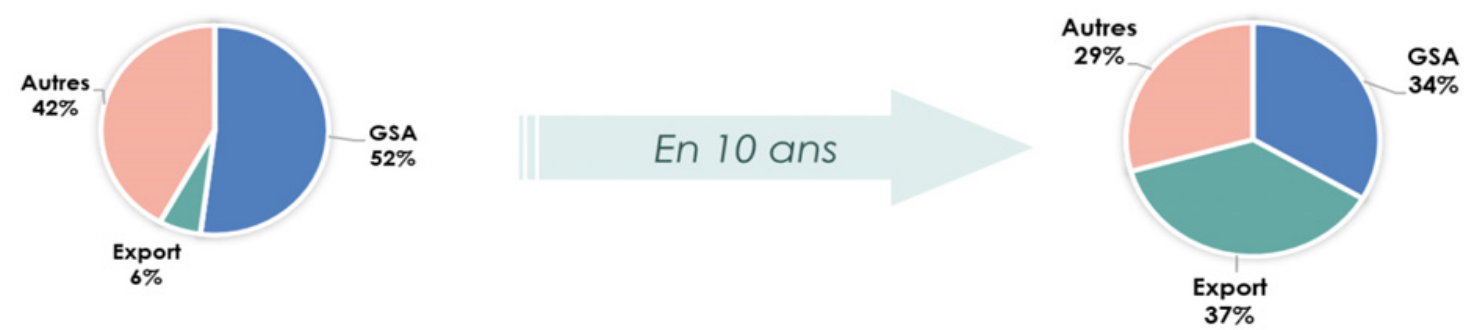

\section{Breakdown in volume of Provence wines exports in the US (CIVP, french customs, in hl)}

$2,50,000$

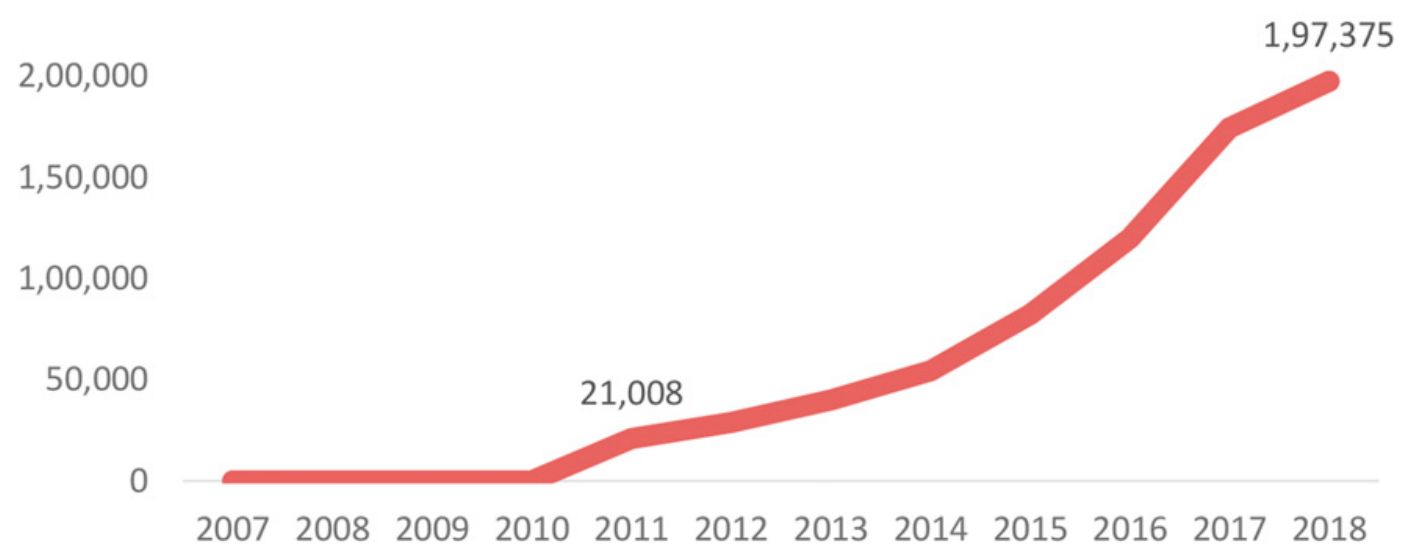

\section{Le consommateur au cœur du succès du rosé !}

Le développement et le succès passé et futur du marché repose évidemment sur les attentes des consommateurs. En effet, dans les 10 dernières années, non seulement le goût des consommateurs a évolué (recherche de boissons moins «lourdes», moins sucrées) mais également le rapport connaissance/plaisir qui a basculé en faveur de second. Les consommateurs recherchent aujourd'hui des produits simples et authentiques. Avec sa couleur pâle, son goût sec, frais et fruité, le vin rosé et en particulier les Vins de Provence ont su parfaitement répondre à l'approche plus décontractée et décomplexée du vin.
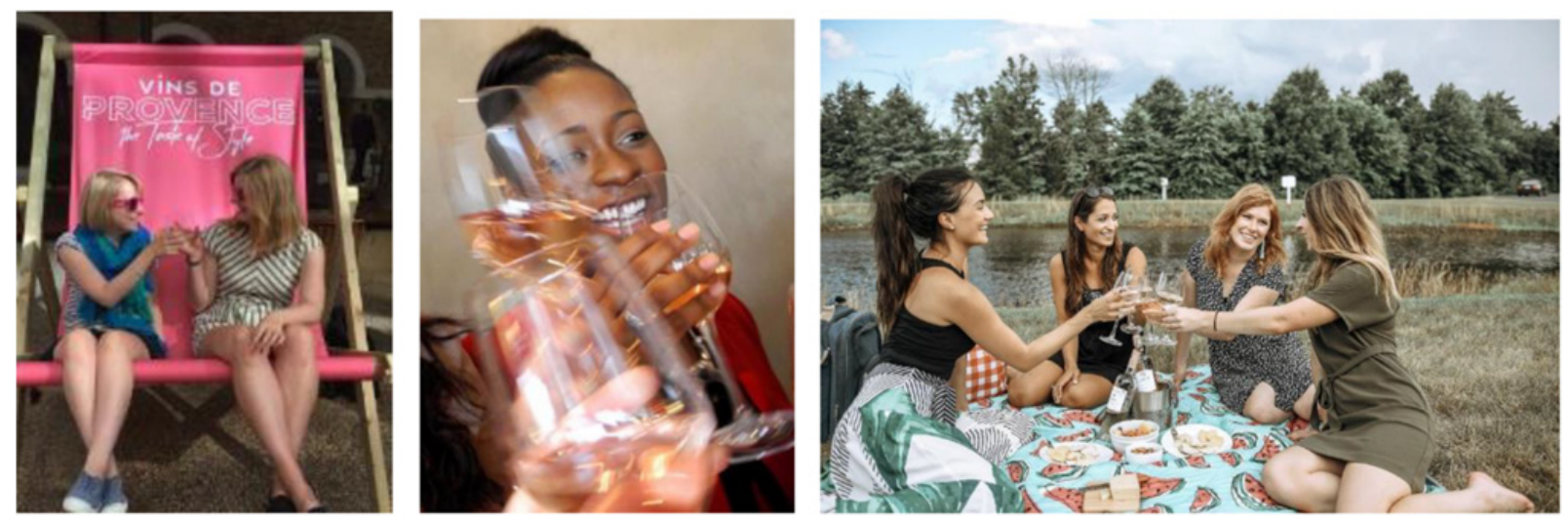

Les Millenials ont joué et joueront un rôle important pour le vin rosé. Cette génération qui a aujourd'hui entre 18 et 35 ans est un moteur de la croissance de la consommation de vins rosés et représentera la plus grosse partie des consommateurs dans le monde d'ici 15 ans. D'un point de vue sociologique, c'est la première génération à avoir un comportement à la fois mondialisé et totalement individualisé. La consonance cognitive et le lien entre ses valeurs et ce qu'elle consomme est essentielle pour cette génération. 
Le rose en général a ainsi un statut particulier : ce n'est pas qu'une couleur, c'est une valeur !

Le vin rosé est ainsi synonyme d'innovation, de jeunesse, de joie de l'instant, de simplicité en opposition à la tradition, au passé, au snobisme...

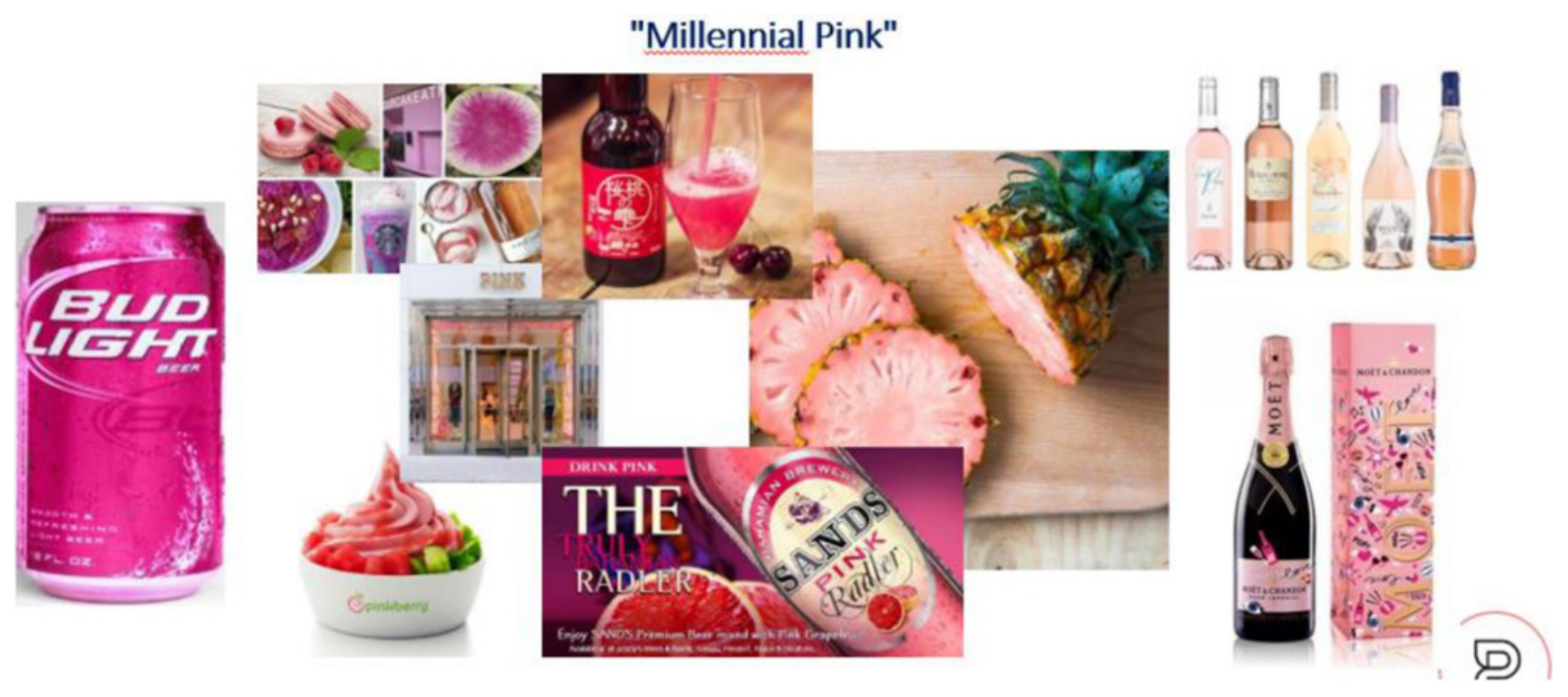

La capacité du vin rosé à rester conforme aux valeurs de cette génération, en particulier au niveau environnemental sera essentiel à l'avenir.

\section{Conclusion}

Le développement potentiel du marché du rosé pose de nombreuses questions. En premier lieu, quelle offre sera proposée pour répondre à la demande ? En dehors de la Provence, peu de vignobles sont spécialisés sur la couleur et gèrent encore la quantité de rosé produit de manière opportuniste. Les vignobles producteurs de rosés sauront-ils également mettre en œuvre des solutions de transition écologique pour atténuer les aléas de production ? En terme de qualité, la demande internationale est axée sur le «Provence style» avec des rosés secs, clairs et aromatiques. Mais est-il opportun d'uniformiser l'offre ? Par ailleurs, le changement climatique d'une part, les attentes des consommateurs d'autre part, ne vont-ils pas faire évoluer le style des vins rosés consommés d'ici 10 ans ? Enfin, l'internationalisation du marché pose le défi du maintien qualitatif et de la réduction de l'impact de l'expédition des vins vers des destinations de plus en plus lointaines.

Sources: Observatoire mondial du rosé France Agrimer/CIVP/Dowel, étude Vinexpo/IWSR, études Wine Intelligence, panel IRI, douanes françaises.
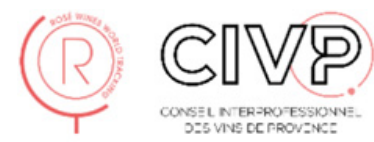

Agrex Consulting
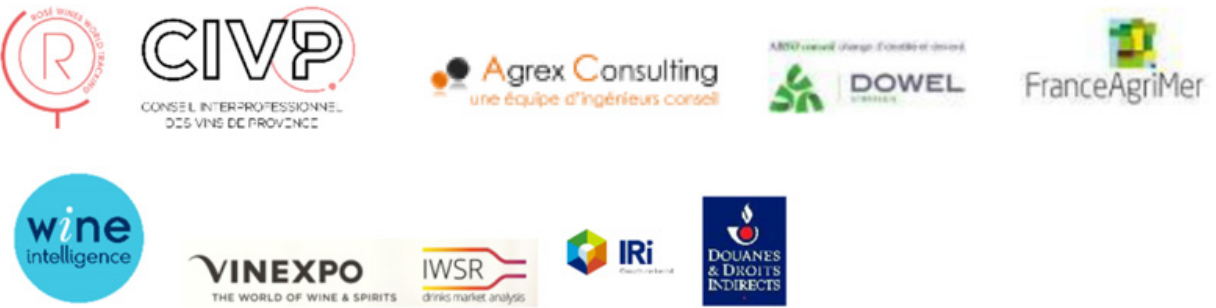

VINEXPO

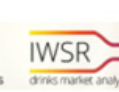

